\title{
El impacto emocional de la pandemia en los jóvenes: Sociabilidad, conflictos, y política
}

\author{
[The emotional impact of the pandemic on young people:
}

\author{
Sociability, conflict, and politics]
}

\author{
Rodrigo Asún 1(D), Irma Palma 1 (D) Roberto Aceituno ${ }^{1}$ (D) y Fabian Duarte ${ }^{1,2}$ (D) \\ ${ }^{1}$ Universidad de Chile; 2 Núcleo Milenio de Desarrollo Social
}

\begin{abstract}
Resumen
Diversos estudios han mostrado que la pandemia del Covid-19 ha tenido diversos efectos sobre la sociedad que sobrepasan en ámbito de la salud física. Como equipo hemos constatado en nuestras investigaciones que uno de los grupos que más han visto afectada su salud mental y bienestar subjetivo por la pandemia han sido los jóvenes. En función de ello, en esta investigación exploramos posibles explicaciones para este mayor impacto, apoyándonos en la sociología de las emociones y en los resultados de una encuesta aplicada en Chile a una muestra de 2,552 personas, de las cuales 629 personas corresponden a jóvenes entre 18 y 29 años. Usando estadística bivariada y análisis de clase latente, encontramos que los jóvenes que han experimentado mayores malestares emocionales durante la pandemia no tienen grandes diferencias socioeconómicas con los otros grupos juveniles, pero si en una peor percepción respecto de su salud en general, mayor percepción de conflictos al interior de su hogar, una experiencia de educación online mucho más negativa y una peor evaluación de la gestión sanitaria del gobierno y de la respuesta de la sociedad ante la pandemia. Estos resultados se discuten a la luz de las características de la juventud actual y de la sociología de las emociones.
\end{abstract}

Palabras clave: impacto emocional, malestar emocional, pandemia, covid-19, jóvenes.

\begin{abstract}
Studies have shown that the Covid-19 pandemic has had various effects on society that go beyond physical health. As a team, we have found in our research that young people are among those groups whose mental health and subjective well-being have been most affected by the pandemic. In this article we explore possible explanations for this greater impact, relying on the sociology of emotions and the results of a survey applied in Chile to a sample of 2,552 people, of whom 629 are young people aged between 18 and 29 years. Using bivariate statistics and latent class analysis, we find that young people who have experienced greater emotional distress during the pandemic do not present marked socioeconomic differences to other youth groups. However, they do report a poorer perception of their general health, a higher level of conflict in their home, a much more negative online education experience, and a poorer evaluation of both the government's management of healthcare and society's response to the pandemic. We discuss these results in light of the characteristics of today's youth and the sociology of emotions.
\end{abstract}

Keywords: emotional impact, emotional distress, pandemic, covid-19, youth.

Contacto: La comunicación sobre este artículo debe ser enviada a Rodrigo Asún, email rasun@uchile.cl

Financiamiento: Este trabajo fue realizado en el marco del proyecto Vida en Pandemia financiado por la Rectoría, Vicerrectoria de Investigación y Desarrollo, y la Dirección de Igualdad de Género de la Universidad de Chile, el Institute for Research in Market Imperfections and Public Policy (MIPP), y de United Nations Educational, Scientific and Cultural Organization (UNESCO). 


\section{INTRODUCCIÓN}

La pandemia del COVID-19 que se ha experimentado el mundo desde 2020 ha sido una tragedia por sus consecuencias sanitarias y económicas, y por su fuerte incidencia en el estado de ánimo y salud mental de la población (Jadoo, 2020). En este artículo nos centraremos en un aspecto específico del impacto de la pandemia sobre la población: su impacto en los sentimientos, sentidos, y emociones experimentadas por las personas; es decir, su impacto subjetivo.

Introducirse en el estudio del impacto de una catástrofe sociosanitaria como la pandemia del Covid-19 a través del estudio de las emociones que ha generado en las personas, pudiera parecer una opción demasiado centrada en el individuo para investigar un fenómeno cuyas principales consecuencias de largo plazo parecen ser predominantemente sociales. Sin embargo, desde hace dos décadas que la sociología de las emociones (Bericat, 2000) viene reivindicando el carácter socialmente construido de las emociones (Scheff, 1997) y ha promovido su estudio como una herramienta importante para comprender la subjetividad de los grupos humanos (Hochschild, 1979). Además, integrar las emociones en modelos explicativos de diversos tipos de comportamientos colectivos ha resultado sumamente productivo, por ejemplo, para entender la conducta política de las personas en su dimensión de participación institucional (Groenendyk, 2011), así como en su participación en protestas o movimientos sociales (Asún et al., 2021; Jasper, 2011).

En consecuencia, estudiar las emociones que ha generado la pandemia constituye una ventana informativa sobre su impacto social que permite también entender el comportamiento presente y futuro de distintos grupos de personas frente a este contexto. Por ello, en esta investigación buscamos responder a las siguientes preguntas: ¿cuál fue el impacto emocional que tuvo la pandemia sobre la población?, ¿sobre qué grupo humano tuvo dicha pandemia un mayor efecto emocional?, y ¿qué variables se asocian y podrían explicar ese mayor impacto? Para responder a ellas, desarrollamos esta investigación en el marco del proyecto "Vida en Pandemia: para un aprendizaje social de impactos y respuestas a la crisis en la vida cotidiana" de la Universidad de Chile. El proyecto completo contempló varias olas de encuestas aplicadas a población habitante en el territorio chileno y tuvo como objetivo conocer el impacto material y subjetivo de la actual epidemia sobre los chilenos y chilenas. La encuesta que usamos en este artículo en específico fue la primera ola de esta investigación y se realizó en un contexto particularmente interesante para estudiar el efecto de la pandemia y de las medidas tomadas para contenerla, pues la producción de los datos tuvo lugar en los momentos en que la primera ola de contagios estaba en su punto más alto, por lo que las diversas medidas de confinamiento social y sanitario se encontraban en su máxima intensidad. A su vez, es interesante recordar que la pandemia golpeó a Chile sólo unos meses después de que el estallido social (Somma, et al., 2020) que mostró la profunda insatisfacción de las personas con la situación social del país, sus élites políticas, y sus instituciones. Por ello, las emociones que se experimentaron durante la primera ola de la pandemia también pueden haber estado influidas 
por ese acontecimiento que amplió la brecha entre las autoridades de gobierno y la población.

\section{Impactos diferenciales de la pandemia}

Actualmente es evidente que la pandemia del COVID-19 no ha sido sólo un problema sanitario, sino también económico, educativo, social y de salud mental (Hernández, 2020). Esto ha abierto paso a la noción de que los grupos humanos más afectados por cada una de estas dimensiones de la pandemia pueden ser distintos entre sí. La investigación epidemiológica muestra que el impacto del Covid-19 sobre la morbilidad y mortalidad de la población es mayor en las poblaciones de mayor edad que poseen algún tipo de comorbilidad (Yupari-Azabache et al., 2021) o menores recursos económicos (Mena et al., 2021). En tanto que los trastornos económicos que provocan las medidas de confinamiento social tienden a impactar más fuertemente a la población de menores niveles de ingreso (Vida en Pandemia, 2020a) y los cambios en las rutinas cotidianas al interior del hogar afectan principalmente a las mujeres (Vida en Pandemia, 2020c); y se ha demostrado que el impacto de la pandemia sobre la salud mental ha sido mayor en las mujeres, pero también a la población joven (Mac-Ginty et al., 2021).

Un estudio sobre el impacto subjetivo del Covid-19 (Vida en Pandemia, 2020b) indica que hay tres variables claves para distinguir los grupos de personas más y menos afectadas por la pandemia: nivel socioeconómico, sexo, y edad de las personas; siendo más importantes las distinciones por edad que las de sexo o las de nivel socioeconómico. Producto de su mayor vulnerabilidad, las personas de menores ingresos son uno de los grupos que han visto más deteriorados sus niveles satisfacción subjetiva con la pandemia. Las mujeres, por su parte, son otro de los grupos que más afectados, ya que el confinamiento ha aumentado su vulnerabilidad a la violencia de género (Acosta, 2020), y ha tensionado los recursos que tienen los hogares para el cuidado y educación de los niños, actividad que en Chile desarrollan principalmente las mujeres (Álvarez et al., 2020). El que estos grupos hayan sido altamente afectados es algo que probablemente los investigadores podrían haber anticipado previamente. Sin embargo, el que las personas jóvenes están también altamente afectadas subjetivamente es algo que resulta al menos llamativo pues en principio, la población joven es la que corre menores riesgos de morbi-mortalidad con el Covid-19. La menor vinculación de la población joven con el mundo del trabajo -dado que, al menos en Chile, una parte importante suelen dedicarse a sus estudios de manera casi exclusiva- les protege de los efectos directos del desempleo, su implicación en las tareas del hogar es menor que la que grupos de mayor edad lo que les protege en parte de la sobrecarga, y han podido mantener en gran medida sus actividades educativas en formato online.

En ese contexto, ¿qué hipótesis se podrían sostener para explicar el alto impacto de la pandemia sobre la salud mental juvenil? Sin perjuicio de otras posibles explicaciones, en este artículo sostenemos y presentamos evidencia respecto a que una de las causas más importantes de los problemas emocionales y de salud mental que experimentan los jóvenes tiene que ver con una ruptura en los patrones de sociabilidad de este grupo. La obligación 
de permanecer mayor tiempo al interior de los hogares y la suspensión de las actividades educativas cara a cara exige a los jóvenes aumentar la interacción intergeneracional con sus padres, abuelos, hermanos pequeños y otros, mientras se restringen las actividades de socialización con sus pares generacionales. En consecuencia, hipotetizamos que desde el punto de vista juvenil la pandemia hace entrar en crisis los patrones generacionalmente endogámicos privilegiados por la sociabilidad juvenil chilena actual.

\section{Ser joven en el Chile del siglo XXI}

Si bien es claro que no existe una sola juventud, sino numerosas juventudes y formas de transitar por esta etapa de la vida en función de las condiciones familiares, sociales, educativas, culturales, y familiares (Duarte, 2000), también es cierto que en las sociedades modernas esta etapa de la vida está asociada a algunas características comunes, dos de las cuales son particularmente importantes para este trabajo. Por un lado, los jóvenes tienden a tener menos lazos con el statu quo imperante en las sociedades a las que se incorporan (Muñoz, 2011). Esto hace más probable que graviten hacia posiciones críticas con el estado actual de sus comunidades (Sandoval \& Carvallo, 2019), especialmente cuando existen narrativas previas que critican algunos aspectos de esas sociedades que son centrales para su propio desarrollo como, por ejemplo, las narrativas en torno a los problemas del sistema educativo (Canales et al., 2020). Por otro lado, la mayor parte de los jóvenes enfrentan en esta etapa el importante desafío de construir una identidad personal y social que los sitúe en una posición social satisfactoria tanto para sí mismos y para quienes los rodean (Margulis \& Urresti, 1996). Esta tarea de construcción identitaria en las sociedades modernas tiende a realizarse en el marco de las relaciones entre pares (Bargsted et al., 2020). Por tanto implica una fuerte inversión de tiempo en la construcción de culturas y visiones de mundo compartidas entre ellos y los diversos subgrupos de pares con quienes interactúan (Zarzuri, 2016).

Una consecuencia de las propiedades que hemos descrito es que quienes viven la juventud en un determinado período social e histórico tienden a constituir una generación (Sandoval \& Carvallo, 2019). Una generación está caracterizada por elementos culturales e ideológicos compartidos por la mayor parte de los distintos grupos y culturas juveniles que se construyen en esos momentos. Otra consecuencia, es que la mayoría de las construcciones culturales e ideológicas de una generación están orientadas hacia producir cambios en las sociedades en las que viven, y a incentivar el involucramiento de los jóvenes en acciones que promuevan dicho cambio social (Bellei \& Cabalín, 2013). Esta orientación general hacia el cambio social puede tomar cualquier dirección ideológica, como fue el caso del Partido Nazi en la Alemania de entreguerras o el de la oposición a la guerra de Vietnam en la guerra fría, ya que quienes participaron en ambos movimientos fueron en su mayoría jóvenes. Sin embargo, en las condiciones predominantes en el Chile del siglo XXI, su alineación mayoritaria tiende a ser crítica de las democracias y élites políticas actuales (Instituto Nacional de la Juventud [INJUV], 2015). 
Por lo anterior, no extraña que hayan sido principalmente las generaciones más jóvenes quienes participaron más activamente en el estallido social chileno (Núcleo De Sociología Contingente [NUDESOC], 2020) y quienes sienten mayor distancia de las principales instituciones sociales, políticas, y de control social de la sociedad chilena. Tampoco resulta sorprendente que, antes del estallido social, es decir, antes de que toda esa energía se canalizara hacia la lucha por el cambio social, la salud mental de los jóvenes estuviera ya disminuida (Baader et al., 2014) producto de las condiciones sociales y educativas que hacían particularmente inciertas sus posibilidades de construir proyectos de vida exitosos y satisfactorios (Canales et al., 2020).

Este proceso de construcción intergrupal de identidades e implicación generacional en el cambio social se ha visto interrumpido por la pandemia y las medidas de confinamiento decretadas por las autoridades. Estas medidas han obligado a los jóvenes a un distanciamiento intrageneracional que dificulta la construcción identitaria y la canalización de energías para luchar por el cambio social. Lo anterior, a nuestro juicio, sería una de las principales explicaciones del alto impacto subjetivo que ha tenido la pandemia en los jóvenes, por lo tanto, conocer las emociones que han experimentado permitirá profundizar en dicho impacto.

\section{Sociología de las emociones y el comportamiento}

Hasta hace pocas décadas, las emociones no jugaban un rol importante en la teoría o en la investigación sociológica. La atención que le habían brindado los autores clásicos de la sociología a las emociones era sólo parcial (Bericat, 2000), pues asumían -explícita o implícitamente- que las emociones eran un fenómeno individual -que era parte del campo de investigación de otras disciplinas como la psicologíao un componente irracional de la conducta que no se relacionaba con la vida social, por lo que se les atribuía poco interés sociológico.

Esta situación comenzó a cambiar en la segunda mitad del siglo XX, producto de los trabajos pioneros de Hochschild (1975), Kemper (1978) y Scheff (1979), quienes instalaron en la sociología la preocupación por las causas y consecuencias sociales de los estados emocionales de las personas. Actualmente ese proceso de instalación de las emociones en esta disciplina ha continuado y se ha profundizado, llegando a aceptarse que juegan un rol crucial en conductas tradicionalmente centrales para el trabajo sociológico como el comportamiento político institucional (Groenendyk, 2011) y no institucional (Flam, 2005). En este proceso, también se ha modificado la concepción de las emociones de parte de la disciplina. Las emociones ya no se consideran un mecanismo irracional u opuesto a nuestras cogniciones, sino más bien un sistema de respuesta complementario y alternativo al razonamiento (Yzerbyt et al., 2003). Se ha comprendido la naturaleza social de las emociones, y se las entiende producto y a su vez causa del comportamiento social. Finalmente, en estos tiempos también ha cambiado la definición de las emociones. Se ha dejado de considerar evidente suponer la existencia de un conjunto reducido de emociones básicas universales (Barrett, 2014), para entender las emociones como un producto de la cultura y las sociedades humanas (Russell, 2009). Por ende el contenido y el número de emociones que 
las sociedades y culturas reconocen como tales varia interculturalmente.

Desde esta perspectiva construccionista social y cognitiva de las emociones (Barrett, 2014), es posible preguntarse por qué es importante estudiarlas para comprender los efectos de la pandemia en las personas y en la sociedad. Para responder a esto, proponemos que es posible pensar las emociones como un sistema de respuesta altamente sensible al medio social en que las personas y grupos se desenvuelven. De esta forma, si una sociedad se ve afectada por un desastre socioambiental (Lavell, 1993) y en ella predominan emociones prosociales de bienestar, como la solidaridad, la esperanza, o el orgullo colectivo, la respuesta de esa sociedad a la crisis será muy distinta a la otra sociedad en la cual predominen las emociones activas de malestar como la rabia o la indignación; u otra sociedad en la que predominen emociones pasivas de malestar como el miedo y la tristeza. Como las emociones predominantes en una sociedad son producto de su vida grupal, la prevalencia de ciertas emociones sobre otras también es un indicador de las propiedades, fracturas, y tensiones que esa sociedad experimenta. Por todo esto, en este trabajo nos preguntamos: ¿qué grupos de jóvenes fueron más afectados emocionalmente por la pandemia?, ¿cuáles fueron sus emociones predominantes? y ¿qué variables podrían explicar ese mayor impacto emocional?

\section{MÉTODO}

El universo del estudio fueron personas mayores de 18 años, habitantes de zonas urbanas y rurales de comunas distribuidas a lo largo de todo el territorio nacional. Con el fin de obtener una muestra heterogénea desde los puntos de vista territorial, social y demográfico, y que además tuviera subgrupos de tamaño suficiente como para realizar análisis comparativos con una adecuada potencia estadística, se decidió fijar cuotas muestrales en función de la región que se habitaba, el sexo, la edad y el nivel socioeconómico de las personas. Los participantes fueron seleccionados por muestreo no probabilístico. Se encuestó a 2,552 personas, de las cuales 558 personas que vivían en las regiones del norte del país (21.9\%), 342 que habitaban las regiones del centro del país (13.4\%), 621 que vivían en las zonas del sur del país (24.3\%) y 1,031 que habitaban la Región Metropolitana (40.4\%). La descripción de la muestra obtenida según el resto de las cuotas se puede observar en la Tabla 1.

El trabajo de campo se realizó por medio de una empresa privada (NETQUEST) experta en la aplicación de encuestas online a paneles de personas previamente reclutadas, y que disponía de un panel de tamaño y heterogeneidad suficiente como para tener la expectativa razonable de obtener una muestra consistente con las cuotas requeridas. Como es habitual en estos sistemas de aplicación de encuestas, quienes respondieron recibieron un pago por parte de la empresa encuestadora. Los datos fueron producidos durante la primera quincena de julio de 2020, período que coincide con 
la fase descendente de la primera ola de contagios de la pandemia del Covid-19 que afectó a Chile, sin embargo, permanecían vigentes las restricciones a la movilidad a lo largo del territorio nacional.

Tabla 1.

Tamaño de la Muestra Según Sexo, Edad, y Grupo Socioeconómico (GSE)

\begin{tabular}{|c|c|c|c|c|c|c|}
\hline \multirow[b]{2}{*}{ GSE } & \multirow[b]{2}{*}{ Sexo } & \multicolumn{4}{|c|}{ Edad } & \multirow{2}{*}{ Total } \\
\hline & & 18 a 29 & 30 a 44 & 45 a 59 & 60 o más & \\
\hline \multirow{2}{*}{ Alto } & Hombre & 26 & 52 & 54 & 110 & 242 \\
\hline & Mujer & 27 & 48 & 52 & 49 & 176 \\
\hline \multirow{2}{*}{ Medio } & Hombre & 115 & 155 & 120 & 174 & 564 \\
\hline & Mujer & 107 & 131 & 173 & 134 & 545 \\
\hline \multirow{2}{*}{ Bajo } & Hombre & 166 & 125 & 93 & 88 & 472 \\
\hline & Mujer & 188 & 114 & 179 & 72 & 553 \\
\hline \multirow{3}{*}{ Total } & Hombre & 307 & 332 & 267 & 372 & 1278 \\
\hline & Mujer & 322 & 293 & 404 & 255 & 1274 \\
\hline & Total & 629 & 625 & 671 & 627 & 2552 \\
\hline
\end{tabular}

El cuestionario aplicado constó de 66 preguntas de respuesta cerrada simple y múltiple, con un tiempo de aplicación promedio de alrededor de 25 a 30 minutos. El cuestionario estaba dividido en una serie de módulos relativamente independientes que tocaban diversos temas relacionados con las condiciones laborales y educativas, distribución de tareas en el hogar, estado de salud, cumplimiento de medidas de cuarentena, emociones y salud mental, entre otros. Muchas de estas preguntas fueron usadas en esta investigación como variables independientes para explicar las emociones experimentadas por las personas en el contexto de la pandemia.

Nuestra variable dependiente fue el ítem: "lo que ha experimentado con más intensidad en las últimas dos semanas, usted diría que es...", seguida de 26 estados emocionales o subjetivos (e.g., esperanza, rabia), frente a cada uno de los cuales las personas debían marcar si habían experimentado ese estado usando cuatro niveles de frecuencia desde "muy frecuentemente" a "nunca".

Los estados emocionales consultados fueron definidos por el equipo de investigación en función de su pertinencia para la situación de pandemia estudiada. Muchos de ellos corresponden a lo que habitualmente se entiende como emociones reflejas (Jasper, 2018), pero en otros, su carácter de emociones podría ser discutible, siendo posible considerarlos más bien estados subjetivos, estados de ánimo, o sentimientos (Jasper, 2018). Pese a lo anterior, apoyándonos en una definición amplia de emociones que incorpora como tales a las emociones reflejas, los estados de ánimo, y los sentimientos (para una descripción detallada de cada una ver: Jasper, 2018), en esta investigación los consideraremos a todos ellos como experiencias emocionales. En términos concretos, las experiencias emocionales por las que preguntamos fueron: aburrimiento, agobio, alegría, angustia, cansancio, confianza, 
confusión, desconfianza, desesperanza, esperanza, frustración, gratitud, inquietud, inseguridad, miedo, optimismo, pesimismo, preocupación, rabia-ira, responsabilidad, seguridad, serenidad, soledad, solidaridad, tranquilidad-calma, y tristeza. Para analizar los datos usamos tres tipos de técnicas estadísticas: (a) estadística descriptiva para caracterizar el estado emocional de los sujetos en el contexto de pandemia; (b) estadística multivariada en la forma de un análisis de clases latentes (Goodman, 2002) para identificar subgrupos de sujetos con distintos niveles de afectación emocional producto de la pandemia; (c) estadística bivariada para explorar otras variables asociadas a la pertenencia a cada uno de los subgrupos identificados por el análisis de clases latentes y que, por lo tanto, permitan construir explicaciones sobre las razones por las cuales algunas personas fueron más o menos afectadas emocionalmente.

\section{RESULTADOS}

En primer lugar, decidimos confirmar que eran los jóvenes los más afectados subjetivamente por la pandemia y las medidas para controlarla. Esto lo hicimos comparando las respuestas de los encuestados de 18 a 29 años con los otros grupos de edad. Encontramos que los jóvenes fueron quienes más reportaron que su salud mental había empeorado desde antes de la pandemia (63.9\% versus $52.7 \%$ en los otros grupos; $\left.\mathrm{X}^{2}(2)=35.4, \mathrm{p}<.001\right)$, que habían tenido problemas para dormir en la última semana ( $54.8 \%$ versus $39.3 \%$ en los otros grupos; $\left.\mathrm{X}^{2}(3)=50.8, \mathrm{p}<.001\right)$, que se sentían desanimados o muy desanimados ( $41.3 \%$ versus $31.2 \%$ en los otros grupos; $\mathrm{X}^{2}(4)=44.5, \mathrm{p}<.001$ ), y que habían sentido miedo frecuente o muy frecuentemente en las dos semanas anteriores a la aplicación de la encuesta (34.6\% versus $27.4 \%$ en los otros grupos; $\mathrm{X}^{2}(4)=22.9$, $\mathrm{p}<.001$ ).

En la Figura 1 podemos observar que al ordenar a hacia la izquierda de la figura los estados emocionales que los adultos sintieron más frecuentemente que los jóvenes y situamos hacia la derecha los que experimentaron más frecuentemente los jóvenes, existen agrupaciones distintas. En los adultos predominan emociones como la serenidad, esperanza, y la tranquilidad, mientras que en los jóvenes predomina el cansancio, el agobio, la frustración, y el aburrimiento. Es interesante notar que este mayor impacto subjetivo de la pandemia no parece deberse a una más alta percepción de riesgo de enfermedad o muerte por parte del Covid-19, pues los jóvenes perciben su salud como buena o bastante buena en mayor medida que los otros grupos $\left(45.8 \%\right.$ versus $39.2 \%$ en las otras edades; $\mathrm{X}^{2}(4)=$ $16.3, \mathrm{p}<.003)$ y evalúan su riesgo de contagiarse como significativamente menor $(28.9 \%$ versus $33.2 \%$ en las otras edades; $\left.\mathrm{X}^{2}(4)=10.5, \mathrm{p}<.032\right)$.

Luego de establecer que fueron los jóvenes quienes se vieron más impactados en su salud mental y estado emocional por la pandemia y las medidas tomadas para controlarla, hicimos un análisis de clase latente con la submuestra de jóvenes de entre 18 y 29 años, para evaluar si era posible distinguir la existencia de grupos de jóvenes más y menos impactados emocionalmente. Este análisis fue realizado usando los 26 estados emocionales que se muestran en la Figura 1. 
Los resultados del análisis de clases latentes muestran que suponer la existencia de cuatro clases latentes no mejora significativamente la predicción de las respuestas de los jóvenes que el suponer tres clases (Loglikelihood $=-16928.4$; Akaike $=34486.8$; BIC $=35886.7$; Vuong-Lo-Mendel-Rubin $=394.9, p=.250 ;$ Lo-Mendel-Rubin Adj. LRT = 865.1, $p=.251)$, mientras que suponer la existencia de tres sí lo hace respecto de la existencia de dos clases (Loglikelihood $=-17361.8 ;$ Akaike $=35195.5 ;$ BIC $=36244.3$, Vuong-Lo-Mendel-Rubin $=193.3, p=$ .004; Lo-Mendel-Rubin Adj. LRT = 1476.1, $p=.004$ ). Por ello, en esta investigación asumiremos la existencia de tres grupos de jóvenes respecto del impacto emocional de la pandemia.

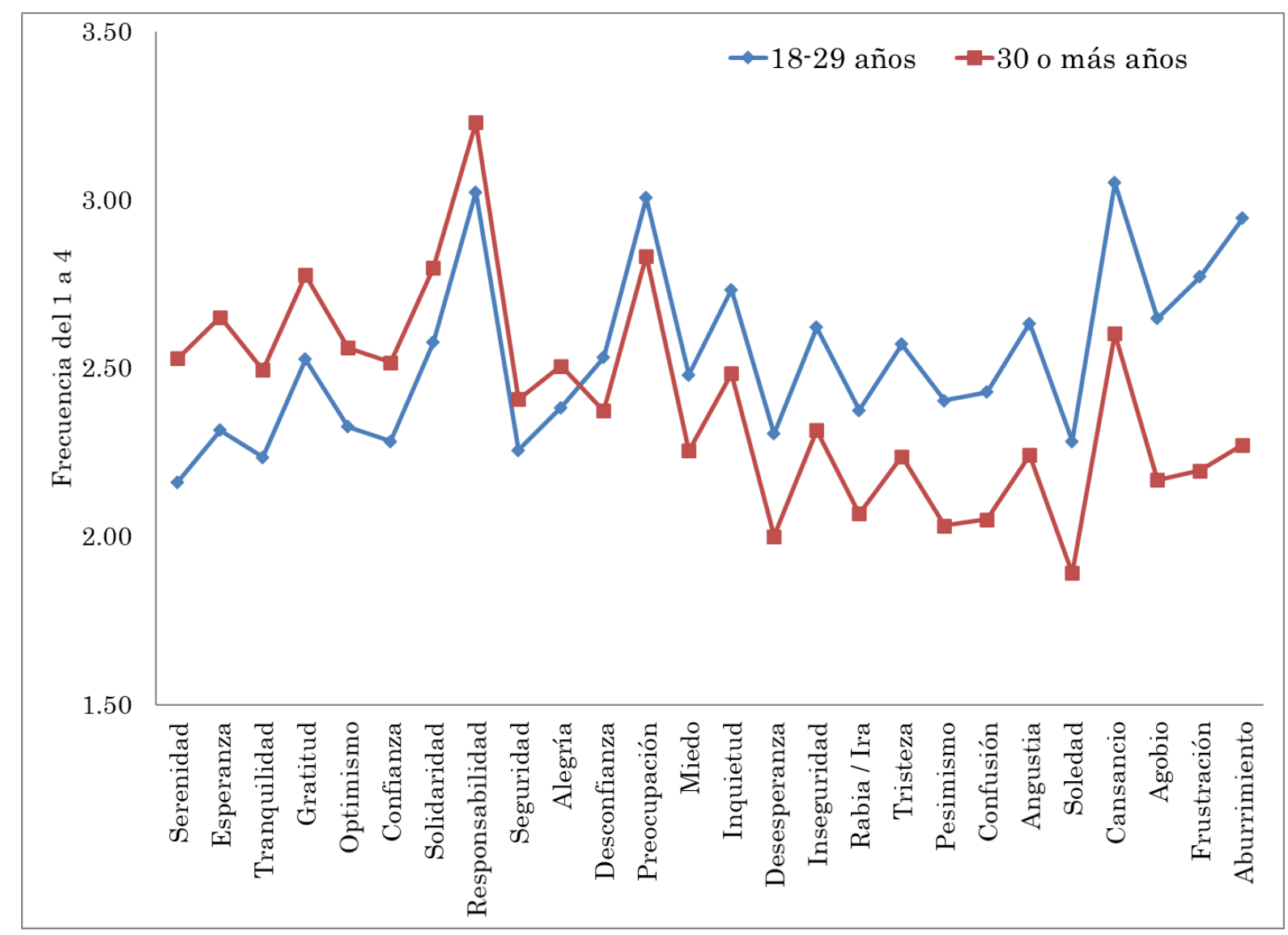

Figura 1. Estados Emocionales Según Edad.

Para describir los tres grupos de jóvenes que emergen al considerar el estado emocional frente a la pandemia hemos construido la Figura 2. En ella podemos observar que el grupo 1 (en color azul y compuesto por 161 jóvenes, es decir el $25.6 \%$ de ellos) son quienes manifiestan estados emocionales de bienestar y menores niveles de malestar emocional. En consecuencia, los denominaremos "poco afectados". Por su parte, el grupo 2 (en color verde y compuesto por 191 jóvenes, es decir el 30,4\% de ellos), está 
integrado por quienes manifiestan estados de bienestar y malestar en casi igual medida, por lo que los denominaremos "medianamente afectados". Finalmente, el grupo 3 (en rojo y compuesto por 277 jóvenes, es decir el 44\% de ellos), está integrado por quienes manifiestan una mucho mayor frecuencia de estados emocionales de malestar respecto de los de bienestar, por lo que los denominaremos "muy afectados". Con la finalidad de comparar, en la Figura 2 se integran las respuestas promedio de los encuestados de 30 años y más (línea punteada), quienes tienden a encontrarse entre el grupo poco afectado y el grupo medianamente afectado, y habitualmente lejos de las respuestas del grupo altamente afectado.

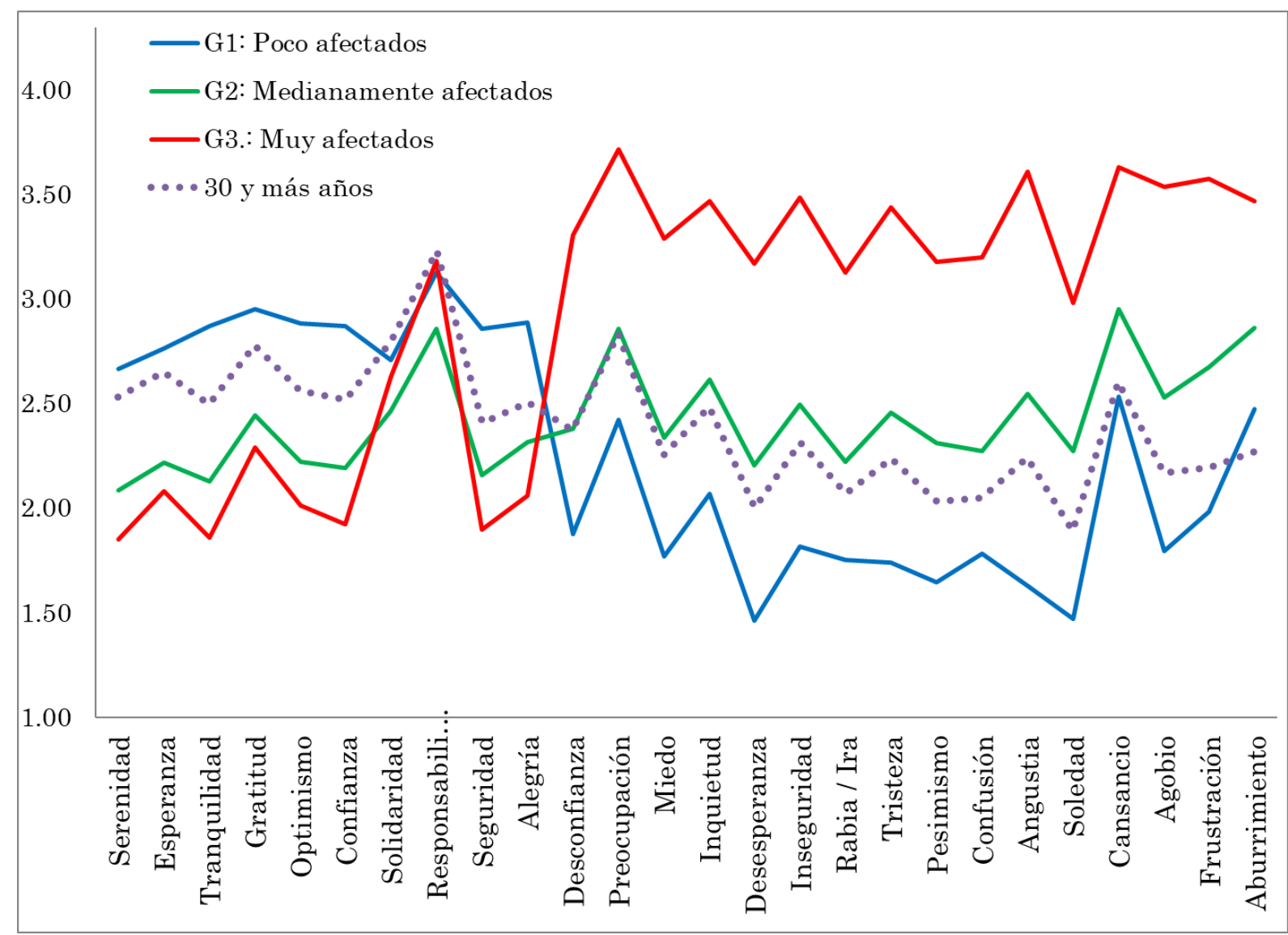

Figura 2. Estados Emocionales en los Grupos de Jóvenes Según su Nivel de Afectación por la Pandemia.

En consecuencia, las diferencias más claras en el nivel de afectación por la pandemia entre los jóvenes y los adultos se encuentran en el grupo de jóvenes muy afectados. Si no existiera este grupo, jóvenes y adultos serían estarían esencialmente igual de afectados por la pandemia. Por lo tanto, si comprendemos que distingue al grupo de jóvenes muy afectados de los otros dos, avanzaremos hacia comprender las causas de que la pandemia y las medidas adoptadas para combatirla hayan impactado más a los jóvenes que a los otros grupos de edad. 
Para confirmar que los grupos detectados función de la experiencia emocional tienen relación con diferentes vivencias subjetivas y estados de salud mental respecto de la pandemia, hicimos una serie de análisis estadísticos que confirmaron que el grupo de los muy afectados era significativamente distinto de los otros dos. Así, mientras el $88.5 \%$ de los integrantes de este último grupo declararon que su salud mental había empeorado, sólo afirmaron eso el $66.4 \%$ de los medianamente afectados y el $30.4 \%$ de los poco afectados $\left(\mathrm{x}^{2}(4)=140.9, \mathrm{p}<.001\right)$. De igual forma, el $57.6 \%$ de los muy afectados reportó problemas para dormir en la última semana, frente a sólo el $24.2 \%$ y el $15.5 \%$ de los medianamente y poco afectados respectivamente. Finalmente, respecto de su percepción de riesgo socio sanitario, el $64.4 \%$ del grupo muy afectado declaró que en las últimas dos semanas había experimentado miedo, frente a sólo el $27.8 \%$ del grupo medianamente afectado y al $11.2 \%$ del poco afectado $\left(\mathrm{X}^{2}(8)=202.4, \mathrm{p}<.001\right)$. Estas diferencias no fueron producto de condiciones demográficas o sociales ya que no se encontraron diferencias significativas entre los tres grupos en relación a las variables sexo, edad, estado civil o tener hijos. Tampoco se encontraron diferencias según región de residencia, estrato socioeconómico, nivel educativo, ni actividad principal. Respecto de esta última variable, los grupos no se diferenciaron en relación a estar estudiando, trabajando o desarrollando ambas actividades. Tampoco hubo diferencias según el tamaño de la vivienda que habitaban ni el número o tipo de personas con las que vivían. La mayor parte de los jóvenes encuestados vivían con su madre, hermanos, y su padre; sólo una minoría vivía solo o con otros miembros de su familia.
Si la heterogeneidad entre los tres grupos no es de carácter sociodemográfico ¿qué los distingue? Los datos indican que las principales diferencias se encuentran en tres ámbitos. En primer lugar, en el ámbito de los miedos sociosanitarios que cada grupo declara experimentar. El grupo muy afectado indica tener un estado de salud un poco más frágil que los otros dos grupos, pues un $34 \%$ de ellos señala que su salud en general es bastante buena o muy buena, frente al 39.8\% de los medianamente afectados, y al $66.5 \%$ de los poco afectados $\left(\mathrm{X}^{2}(8)=65.1, \mathrm{p}<.001\right)$. Posiblemente esta fragilidad en su salud percibida lleva al grupo muy afectado a evaluar como tendencialmente mayor su riesgo de contagiarse durante la pandemia, pues el $35.6 \%$ declara sentirse en riesgo alto o muy alto, frente al $27.4 \%$ de los medianamente afectados, $\mathrm{y}$ al $23.6 \%$ de los poco afectados $\left(\mathrm{X}^{2}(8)=15.3, \mathrm{p}=.054\right)$. No obstante, los miedos que más diferencian a estos grupos no son sólo los sanitarios, sino también los socioeconómicos. Al mismo tiempo que el grupo muy afectado es quien manifiesta un mayor miedo a la enfermedad de un ser querido $(51.1 \%$ de los muy afectados comparado con el $38.8 \%$ de los medianamente afectados y el $32.5 \%$ de los poco afectados; $\left.\mathrm{X}^{2}(2)=11.7, \mathrm{p}=.003\right)$, también son quienes declaran tener mayor miedo a la pérdida de trabajos de los miembros de su familia $(37.8 \%$ de los muy afectados, frente al $25.1 \%$, y al $21.7 \%$ de los medianamente y poco afectados respectivamente), y a no tener recursos para los gastos indispensables ( $43.3 \%$ de los muy afectados, frente al $32.3 \%$ de los medianamente afectados y al $21.7 \%$ de los poco afectados).

El segundo ámbito en el que se diferencian los grupos de jóvenes es la percepción de conflictos 
al interior de cada familia. Quienes integran el grupo muy afectado son quienes han percibido conflictos más frecuentes al interior de sus hogares durante las dos últimas semanas (51.7\% de los muy afectados frente al $28.8 \%$ y el $30.3 \%$ de los medianamente poco afectados; $X^{2}(8)=48.9$, $\mathrm{p}<.001$ ). Para el grupo muy afectado estos conflictos no sólo son frecuentes, sino que además han aumentado en frecuencia respecto de la situación previa a la pandemia (así lo señala el $61.6 \%$ de este grupo, frente al $49.5 \%$ y al $39.3 \%$ los otros dos grupos; $\left.\mathrm{X}^{2}(4)=15.5, \mathrm{p}=.004\right)$. Además, estos conflictos son evaluados como más intensos por el grupo muy afectado que por los otros dos grupos (29.9\% de los muy afectados califica los conflictos ocurridos como graves o muy graves, frente al $17.5 \%$ y el $16.8 \%$ de los medianamente y poco afectados; $X^{2}(8)=27$, $\mathrm{p}<.001$ ). Es interesante notar que esta mayor percepción de conflictos intrafamiliares luego de la pandemia que reporta el grupo muy afectado, no se vincula con ningún tipo de relación particular entre los miembros de la familia. Así por ejemplo, no se encontraron diferencias significativas entre los grupos de jóvenes y el nivel de conflictos reportados con sus madres, padres, hermanos, hijos, entre la pareja, o con otros familiares. Por ello, para profundizar en la naturaleza de esos conflictos tendremos que avanzar hacia la tercera diferencia que existe entre los grupos de jóvenes diversamente afectados por la pandemia.

La Tabla 2 resume los resultados del tercer ámbito que diferencia entre los grupos de jóvenes: su vivencia subjetiva de la educación online. En la tabla podemos ver claramente que para el grupo muy afectado la educación online ha resultado una experiencia mucho más negativa que para los otros dos grupos, destacando especialmente la alta distancia en el grado de estrés que les parece producir, resultado que es una de las diferencias estadísticas más fuertes de este estudio. .

\section{Tabla 2.}

Experiencia educativa online según grupos de jóvenes

\begin{tabular}{lccc}
\hline \multirow{2}{*}{ Preguntas } & \multicolumn{3}{c}{ Grupos de jóvenes } \\
\cline { 2 - 4 } & Muy afectados & $\begin{array}{c}\text { Medianamente } \\
\text { afectados }\end{array}$ & $\begin{array}{c}\text { Poco } \\
\text { afectados }\end{array}$ \\
\hline Me perturba mucho estudiar desde casa. & $75.4 \%$ & $42.1 \%$ & $38.7 \%$ \\
Es displacentero estudiar desde su casa & $71.4 \%$ & $49.1 \%$ & $59.2 \%$ \\
Es muy estresante estudiar desde su casa & $83.1 \%$ & $36.0 \%$ & $26.5 \%$ \\
\hline
\end{tabular}

Nota: Todas las diferencias del grupo muy afectado respecto de los otros dos grupos son estadísticamente significativas a un $99.9 \%$ de confianza.

Este mayor estrés en la experiencia educativa no parece estar relacionado o acrecentado por haber tenido que hacerse cargo de tareas de cuidado al interior del hogar, puesto que no hay diferencias significativas entre los grupos de jóvenes en relación con haber aumentado durante la pandemia el tiempo que dedican a cuidar niños, adultos mayores, o personas con algún tipo de discapacidad. Sin embargo, este mayor estrés educativo sí podría estar relacionado con las tareas 
reproductivas al interior del hogar, puesto que los jóvenes muy afectados por la pandemia declararon estar dedicando mucho más tiempo que antes a cocinar, hacer limpieza y a otras actividades similares en sus casas $(55.5 \%$ del grupo muy afectado, frente al $35.7 \%$ y el $35 \%$ de los otros grupos).

Finalmente, nos preguntamos respecto de las diferentes percepciones políticas y sociales que pudieran tener aquellos jóvenes más impactados por la pandemia, respecto del resto. En la Tabla 3 podemos observar que el grupo de jóvenes muy afectados fueron quienes manifestaron las peores evaluaciones de la gestión del gobierno en su confiabilidad, efectividad, veracidad, y orientación hacia la protección de las personas más vulnerables. En otras palabras, hay una interrelación entre el impacto emocional de la pandemia y la evaluación de un gobierno que parece no haber sido capaz de controlarla.

Tabla 3.

Evaluación del Gobierno Según Grupos de Jóvenes

El gobierno ha sido...

\begin{tabular}{ccc} 
Grupos de jóvenes \\
\hline $\begin{array}{c}\text { Muy } \\
\text { afectados }\end{array}$ & $\begin{array}{c}\text { Medianamente } \\
\text { afectados }\end{array}$ & $\begin{array}{c}\text { Poco } \\
\text { afectados }\end{array}$ \\
$59.2 \%$ & $40.8 \%$ & $46.6 \%$ \\
$53.4 \%$ & $31.0 \%$ & $37.9 \%$ \\
$50.8 \%$ & $27.8 \%$ & $33.5 \%$ \\
$44.0 \%$ & $19.9 \%$ & $25.5 \%$ \\
\hline
\end{tabular}

Nada confiable en su manejo de la pandemia.

Nada efectivo en su manejo de la pandemia.

Nada verídico en sus informaciones sobre la pandemia.

Nada orientado a la protección de los más vulnerables.

Nota: Todas las diferencias del grupo muy afectado respecto de los otros dos grupos son estadísticamente significativas a un $99.9 \%$ de confianza.

Sin embargo, la Tabla 4 muestra que, en términos de evaluaciones políticas y sociales, el grupo de los jóvenes muy afectados no limita sus críticas al gobierno, sino que las extiende a la sociedad, la que no parece haberse comportado como ellos hubieran esperado, pudiendo suponerse que la hacen al menos parcialmente responsable de la magnitud de los efectos sanitarios y sociales de la pandemia. En el grupo más afectado por la pandemia aparecen opiniones ambiguas hacia las restricciones que se han tomado para combatir los contagios. Por un lado, los muy afectados son quienes se manifiestan más claramente de acuerdo con la prioridad de la salud sobre la economía al momento de decidir qué medidas se deben adoptar para controlar la pandemia, pero también son el grupo que más se manifiesta de acuerdo con que esas medidas fueron exageradas, generando pobreza (ver Tabla 4). En otras palabras, se trata de un grupo heterogéneo en este punto. Si bien una mayoría optaría por medidas más restrictivas, una minoría tomaría la opción opuesta. Se podría hipotetizar que lo que une a ambos extremos es la demanda por medidas que sean efectivas y permitan volver a la normalidad lo antes posible.

Es interesante notar que, entre la crítica a un gobierno y una sociedad ausentes, y a las medidas poco efectivas para sobrellevar la pandemia, un grupo minoritario de jóvenes saca lecciones 
individualistas. Ante la falta de apoyo desde las estructuras políticas o sociales, para algunos de ellos la solución es familiar o individual: cada familia e individuo debe enfrentar las catástrofes siguiendo sus propios lineamientos. Desconocemos si esta opción individualista es producto de una socialización previa propia en el marco de una sociedad neoliberal avanzada, como ha sido habitualmente calificado Chile (Maza \& López, 2018), o ha sido un aprendizaje obtenido a partir de lo que han experimentado durante esta pandemia, pero resulta preocupante hacia el futuro que esa sea una de las enseñanzas que postule uno de los grupos más impactados crisis sanitaria y social que estamos viviendo.

Tabla 4.

Opiniones Sobre la Sociedad Según Grupos de Jóvenes

¿Qué opinas respecto de las siguientes frases?

(\% indica "muy de acuerdo")

Las personas no se han tomado a este virus tan en serio como se necesita.

No he sentido que en esta pandemia haya la solidaridad y cooperación que ocurre en otros casos.

Hay que proteger la salud de la población aunque eso genere problemas a la economía.

La salud es muy importante, pero las medidas que se tomaron fueron exageradas, generando pobreza.

Un aprendizaje de esta pandemia es que cada persona y familia debe prepararse por su cuenta para afrontarla.

Yo habría preferido que cada uno hubiera podido decidir libremente como luchar contra este virus.

\begin{tabular}{ccc}
\multicolumn{3}{c}{ Grupos de jóvenes } \\
$\begin{array}{c}\text { Muy } \\
\text { afectados }\end{array}$ & $\begin{array}{c}\text { Medianamente } \\
\text { afectados }\end{array}$ & $\begin{array}{c}\text { Poco } \\
\text { afectados }\end{array}$ \\
\hline $61.8 \%$ & $46,2 \%$ & $47,8 \%$ \\
$30.4 \%$ & $13.7 \%$ & $14.3 \%$ \\
$52.9 \%$ & $39.4 \%$ & $43.5 \%$ \\
$12.6 \%$ & $5.4 \%$ & $8.1 \%$ \\
$34.6 \%$ & $14.8 \%$ & $21.1 \%$ \\
$11 \%$ & $2.9 \%$ & $5.6 \%$
\end{tabular}

Nota: Todas las diferencias del grupo muy afectado respecto de los otros dos grupos son estadísticamente significativas a un $99.9 \%$ de confianza.

\section{CONCLUSIONES}

El principal resultado de la presente investigación ha sido el develar algunas de las razones por las cuales los jóvenes son los más afectados por la actual pandemia en su salud mental, vida emocional, y subjetividad. Estas razones tienen que ver con que, por muy importantes que sean los riesgos sanitarios en mortalidad y morbilidad del Covid-19, uno de sus impactos negativos más perdurables fueron justamente las medidas de distanciamiento social adoptadas para contener la pandemia. Quizás eso sea lo que explique que el grupo etario que se ha visto emocionalmente menos afectado por la pandemia haya sido el de los adultos mayores (Vida en Pandemia, 2020b), que constituyen el grupo más vulnerable desde un punto de vista sanitario. Sin embargo, se podría hipotetizar que dicho grupo, ha recibido más atención y apoyo que ningún otro sector de la población durante este periodo, lo que 
posiblemente contrasta con el relativo abandono en que se encuentran habitualmente los adultos mayores (Gempp \& Benadof, 2017), produciéndose el paradójico efecto de que la pandemia mejora, o al menos no empeora, la experiencia emocional de quienes están más vitalmente amenazados por la enfermedad. Con relación a los adultos, la mayor parte de ellos viven con las familias que han constituido $\mathrm{y}$, por tanto, su bienestar no depende tanto de la interacción con personas externas a sus familias (e.g., amigos, colegas, vecinos), sino de la buena marcha de su núcleo familiar. Sobre este grupo la pandemia ha impactado con fuerza principalmente al hacer inestables las fuentes laborales, además de obligar a una vida más intensa al interior de las viviendas, producto del cierre de los colegios y del traslado al hogar de muchas actividades laborales. Esto afecta especialmente a las mujeres que son madres con hijos en edad escolar, que ven así aumentadas sus responsabilidades, dada la desigual distribución de las labores de reproducción y cuidado al interior del hogar en Chile (Álvarez et al., 2020). Sin embargo, el impacto del distanciamiento social sobre este grupo no parece haber sido tan intenso, posiblemente producto de que la mayor parte de las interacciones emocionalmente significativas de estas personas ocurren al interior de sus viviendas. Además, es razonable suponer que el desarrollo de nuevas habilidades de comunicación online en este grupo etario ha tendido a compensar la pérdida de contactos cara a cara con personas significativas de fuera del hogar.

Una situación muy distinta es la que viven los jóvenes, que han visto lesionadas sus relaciones afectivas significativas, como son las que desarrollan con sus pares generacionales (Bargsted et al., 2020). Ello no sólo ha sido consecuencia de las medidas de distanciamiento social general, sino también del cierre de los establecimientos educativos, los que constituyen desde su punto de vista no sólo un espacio educativo, sino un espacio de interacción social y de construcción identitaria y biográfica (Núñez \& Litichever, 2016) que se ha visto limitado al trasladarse al mundo online. Producto de la centralidad de la vida escolar para la sociabilidad juvenil, la experiencia educativa a distancia ha sido experimentada por ellos con tanto estrés y desagrado. A esto se agrega que las medidas de confinamiento social y cierre de los colegios y universidades han obligado a los jóvenes a desarrollar una vida más intensa y con más responsabilidades al interior de sus hogares -especialmente en tareas de reproducción, ligadas a la limpieza y alimentación de los habitantes del hogar-lo que aumenta las posibilidades de conflictos dentro de la vivienda. Estos conflictos no parecen estar focalizados en ninguna relación social en particular, sino tratarse de un aumento general de la tensión producto de una intensificación inesperada de la densidad de contactos sociales al interior del hogar. Por otro lado, a diferencia de los grupos adultos -para quienes las posibilidades de la comunicación online fue posiblemente un descubrimiento compensador del distanciamiento social-para los más jóvenes es muy probable que este efecto no haya ocurrido, pues ellos tenían previamente mayor familiaridad y uso de estos medios, con lo que la pérdida de las interacciones cara a cara no fue compensada por una mayor intensificación de la vida online. También es interesante constatar que, pese a la habitualidad 
de esas comunicaciones en la vida de los más jóvenes, el impacto que ha tenido en ellos la pandemia demuestra -a modo de experimento natural-que los contactos cara a cara son críticos para su adecuado desarrollo afectivo, ocupando las vivencias online un rol más bien complementario, lo que confirma algunas investigaciones previas sobre el tema (Winocur, 2006). Respecto del impacto político de la pandemia sobre el grupo de jóvenes más afectados por esta, no sorprende que ellos sean los más críticos con el gobierno, puesto que fueron los jóvenes quienes participaron principalmente en el estallido social (Núcleo De Sociología Contingente [NUDESOC], 2020). No obstante, si es destacable la crítica que hacen a la sociedad y la deriva individualista que proponen algunos sectores juveniles frente a esta carencia de apoyos políticos o sociales. El instrumento usado en este estudio no nos permite saber si esta salida aislacionista existía previamente o es producto de las vivencias que este grupo ha tenido durante la pandemia, pero sin duda resulta preocupante y digna de mayor estudio.

En términos más generales, ha resultado sorprendente que la distinción entre los jóvenes más afectados y menos afectados por la pandemia casi no haya tenido ninguna relación con sus condiciones materiales de vida o sus características demográficas, sino con la forma en que ellos experimentan las restricciones sociales que genera el enfrentamiento de la pandemia. Ello nos lleva a argumentar que este caso es una evidencia de que no siempre la forma en que distintos grupos sociales reaccionan a su medio social, cultural, o económico depende de los medios de vida que tengan a su disposición, sino que en algunas ocasiones al menos habrá otras variables subjetivas o relacionadas con la sociabilidad que serán tanto o más importantes para explicarla. Finalmente, creemos que haber focalizado nuestro estudio en las vivencias emocionales de las personas ha mostrado la potencia de estas últimas para comprender la vida social actual. Las emociones constituyen un sistema de alta sensibilidad y diversidad que permite detectar cambios tempranos en la forma en que las personas están experimentando su vida social y, por tanto, nos ayudan a entender cómo perciben su mundo y como podrían comportarse en el futuro. Por ello, consideramos que profundizar en la sociología de las emociones es un camino interesante y prometedor para esta disciplina en el siglo XXI.

\section{REFERENCIAS}

Acosta, M. L. (2020). Violencia de género en tiempos de pandemia y confinamiento. Revista Española de Medicina Legal, 46(3), 139-145. http://doi.org/10.1016/j.reml.2020.05.005

Álvarez, D., Guerra, C., Tapia, K., \& Varas, G. (2020). Compatibilizando estudios y familia: Ser estudiante y a la vez madre o padre.
Revista de Sociología, 35(2), 61-77. https://doi.org/0.5354/0719-529X.2020.58647

Asún, R. A., Rdz-Navarro, K., Zúñiga, C., \& Louis, W. (2020). Modelling the mediating effect of multiple emotions in a cycle of territorial protests. Social Movement Studies, 1-19. https://doi.org/10.1080/14742837.2020.1867093 
Baader, T., Rojas, C., Molina, J. L., Gotelli, M., Alamo, C., Fierro, C., Venezian, S., \& Dittus, P. (2014). Diagnóstico de la prevalencia de trastornos de la salud mental en estudiantes universitarios y los factores de riesgo emocionales asociados. Revista Chilena de Neuro-Psiquiatría, $\quad$ 52(3), 167-176. http://doi.org/10.4067/S0717-92272014000300004

Bargsted, M., Espinoza, V., \& Plaza, A. (2020). Pautas de homofilia en Chile. Papers. Revista de Sociología, 105(4), 583-612.

https://doi.org/10.5565/rev/papers.2617

Barrett, L. F. (2014). The conceptual act theory: A précis. Emotion Review, 6(4), 292-297. https://doi.org/10.1177\%2F1754073914534479

Bellei, C. \& Cabalín, C. (2013). Chilean student movements: Sustained struggle to transform a market-oriented educational system. Current Issues in Comparative Education, 15(2), 108-123. https://www.tc.columbia.edu/cice/pdf/28175 _15_02_Bellei_Cabalin.pdf

Bericat, E. (2000). La sociología de la emoción y la emoción en la sociología. Papers. Revista de Sociología, 62(1), 145-176. https://doi.org/ 10.5565/rev/papers/v62n0.1070

Canales, M., Guajardo, F., \& Orellana, V. (2020). La elite del llano: De la promesa a las desilusiones en la trayectoria postsecundaria de los jóvenes de la nueva clase media. Última Década, 28(53), 78-102. http://doi.org/10.4067/S071822362020000100078

Duarte, C. (2000). ¿Juventud o juventudes?: Acerca de cómo mirar y remirar a las juventudes de nuestro continente. Última Década, \&(13), 59-77. http://doi.org/10.4067/S0718-22362000000200004
Flam, H. (2005). Emotions' map: A research agenda. En H. Flam \& D. King (Eds.), Emotions and social movements (pp. 19-40). Routledge.

Gempp, L., \& Benadof, D. (2017). Percepción de apoyo familiar de adultos mayores residentes en establecimientos de larga estadía. Revista Chilena de Salud Pública, 21(2), 142-149. https://doi.org/10.5354/0719-5281.2017.48911

Goodman, L. (2002). Latent class analysis: The empirical study of latent types, latent variables, and latent structures, and some notes on the history of this subject. En J. A. Hagenaars \& A. L. McCutcheon (Eds.), Applied latent class analysis (pp. 3-55). Cambridge University Press. Groenendyk, E. (2011). Current emotion research in political science: How emotions help democracy overcome its collective action problem. Emotion Review, 3(4), 455-463. https://doi.org/10.1177\%2F1754073911410746

Hernández, J. (2020). Impacto de la COVID-19 sobre la salud mental de las personas. Medicentro Electrónica, 24(3), 578-594. https://pesquisa.bvsalud.org/portal/resource /pt/biblio-1125017

Hochschild, A. R. (1975). The sociology of feeling and emotion: Selected possibilities. En M. Millman \& R. M. Kanter (Eds.), Another voice. Feminist perspectives on social life and social science (pp. 280-307). Anchor Books.

Hochschild, A. R. (1979). Emotion work, feeling rules and social structure. American Journal of Sociology, 85(3), 551-575. https://doi.org/ $10.1086 / 227049$

Instituto Nacional de Juventud. (2015). Octava encuesta nacional de juventud. https://www. injuv.gob.cl/sites/default/files/8va_enj_2015.pdf 
Jadoo, S. A. A. (2020). COVID-19 pandemic is a worldwide typical biopsychosocial crisis. Journal of Ideas in Health, 3(2), 152-154. https://doi.org/10.47108/jidhealth.Vol3.Iss2.58

Jasper, J. M. (2011). Emotions and social movements: Twenty years of theory and research. Annual Review of Sociology, 37(1), 285-303. https://doi.org/10.1146/annurev-soc-081309-150015

Jasper, J. M. (2018). The emotions of protest. University of Chicago Press.

Kemper, T. (1978). Toward a sociology of emotions: Some problems and some solutions. The American Sociologist, 13(1), 30-41.

https://www.jstor.org/stable/27702310

Lavell, T. A. (1993). Ciencias sociales y desastres naturales en América Latina: Un encuentro inconcluso. Revista EURE - Revista de Estudios Urbano Regionales, 19(58), 73-84.

https://www.eure.cl/index.php/eure/article/view/1121

Mac-Ginty, S., Jiménez-Molina, Á., \& Martínez, V. (2021). Impacto de la pandemia por COVID-19 en la salud mental de estudiantes universitarios en Chile. Revista Chilena de Psiquiatría y Neurología de la Infancia y Adolescencia, 32(1), 23-37. https://www.sopnia.com/noticias/ revistas/vol-no32n1/

Margulis, M., \& Urresti, M. (1996). La juventud es más que una palabra. En M. Margulis (Ed.), La juventud es más que una palabra. Ensayos sobre cultura y juventud (pp. 13-30). Biblos.

Maza, A., \& López, C. (2018). Tensiones prácticas e institucionales en el campo de las ciencias sociales en el Chile del neoliberalismo avanzado: Un estudio cualitativo. Estudios
Nueva Economía, 5(1), 21-40.

https://bit.ly/MazayLopez2018

Mena, G. E., Martínez, P. P., Mahmud, A. S., Marquet, P. A., Buckee, C. O., \& Santillana, M. (2021). Socioeconomic status determines COVID-19 incidence and related mortality in Santiago, Chile. Science, 372(6545), Artículo eabg5298. https://doi.org/10.1126/science.abg5298

Muñoz, V. (2011). Juventud y política en Chile. Hacia un enfoque generacional. Última Década, 19(35), 113-141.https://doi.org/10.4067/S071822362011000200006

Núcleo De Sociología Contingente. (2020). Encuesta Zona Cero [Informe resultados oficial]. https://nudesoc.cl/wp-content/uploads/2020/03/ Informe-Resultados-OFICIAL.pdf

Núñez, P., \& Litichever, L. (2016). Ser joven en la escuela: Temporalidades y sentidos de la experiencia escolar en la Argentina. Psicoperspectivas, 15(2), 90-101. https://doi.org/10.5027/psicop erspectivas-Vol15-Issue2-fulltext-854

Russell, J. A. (2009). Emotion, core affect, and psychological construction. Cognition and Emotion, 23(7), 1259-1283. https://doi.org/10.1080/02699930902809375

Sandoval J., \& Carvallo, V. (2019). Una generación «sin miedo»: Análisis de discurso de jóvenes protagonistas del movimiento estudiantil chileno. Última Década, 27(51), 225-257. http://doi.org/10.4067/S0718-22362019000100225

Scheff, T. J. (1997). Emotions, the social bond, and human reality. Cambridge University Press.

Scheff, T. J. (1979). Catharsis in healing, ritual and drama. University of California Press. 
Somma, N. M., Bargsted, M., Disi Pavlic, R., \& Medel, R. M. (2020). No water in the oasis: The Chilean Spring of 2019-2020. Social Movement Studies, 20(4), 495-502.

https://doi.org/10.1080/14742837.2020.1727737

Vida en Pandemia (2020a). Desestabilización, fragilidad e incertidumbre económica, $y$ crisis del estado de ánimo al vivir en la pandemia [Primer informe de primeros resultados].

http://vidaenpandemia.cl/2020/08/15/reporte1/

Vida en Pandemia (2020b). Modos de sentir: experiencia de la vida cotidiana en pandemia. [Segundo informe de primeros resultados encuesta primera ola].

http://vidaenpandemia.cl/2020/08/15/reporte-2/

Vida en Pandemia (2020c). Desestabilización y reorganización de la vida doméstica durante la crisis del covid-19. [Cuarto informe de primeros resultados].

http://vidaenpandemia.cl/2020/09/01/reporte-4/

Winocur, R. (2006). Internet en la vida cotidiana de los jóvenes. Revista Mexicana de Sociología, 68(3), 551-580.

http://www.scielo.org.mx/scielo.php?script=s ci_arttext\&pid=S0188-25032006000300005
Yupari-Azabache, I., Bardales-Aguirre, L., Rodriguez-Azabache, J., Barros-Sevillano, J. S., \& Rodríguez-Diaz, Á. (2021). Factores de riesgo de mortalidad por COVID-19 en pacientes hospitalizados: Un modelo de regresión logística. Revista de la Facultad de Medicina Humana, 21(1), 19-27.

http://doi.org/10.25176/rfmh.v21i1.3264

Yzerbyt, V., Dumont, M., Wigboldus, D., \& Gordijn, E. (2003). I feel for us: The impact of categorization and identification on emotions and action tendencies. British Journal of Social Psychology, 42(4), 533-549. https://doi.org/10.1348/014466603322595266

Zarzuri, R. (2016). Las transformaciones en la participación política de los jóvenes en el Chile actual. En M. A. Garretón (Coord.), La gran ruptura. Institucionalidad política $y$ actores sociales en el Chile del siglo XXI (pp. 133-159). LOM.
Manuscrito recibido: 02-05-2021 Manuscrito aceptado: 10-07-2021 